\title{
OS NÚCLEOS VASOMOTORES DO BULBO E A REGULAÇÃO CARDIOVASCULAR: NOVAS EVIDÊNCIAS E NOVAS QUESTÕES
}

\author{
VENTROLATERAL MEDULLA AND THE CARDIOVASCULAR CONTROL: NEW EVIDENCE NEW DOUBTS
}

Sergio L Cravo ${ }^{1}$, Daniel A Rosa², Fernanda Kalassa², Willian S Korim², Julia M Hinrichs ${ }^{3}$, Marcos L Ferreira-Neto², Luciana R Di Mônaco², Gustavo R Pedrino²

\begin{abstract}
${ }^{1}$ Docente. ${ }^{2}$ Alunos da Pós-graduação. ${ }^{3}$ Aluna Bolsista de Iniciação Científica. Departamento de Fisiologia. Escola Paulista de Medicina -UNIFESP

CoRRespondênCIA: Dr. Sergio L. Cravo

Disciplina de Fisiologia Cardiovascular. Departamento de Fisiologia. Escola Paulista de Medicina - UNIFESP. Rua Botucatu, 862 CEP 04023-062 São Paulo SP - Brasil - FONE: 5576-4536 - FAX: 5573-7820

Email: sldcravo@fcr.epm.br
\end{abstract}

Cravo SL, Rosa DA, Kalassa F, Korim WS, Hinrichs JM, Ferreira-Neto ML, Di Mônaco LR, Pedrino GR. Os núcleos vasomotores do bulbo e a regulação cardiovascular: novas evidências e novas questões. Medicina (Ribeirão Preto) 2006; 39 (1): 89-100.

RESUMO: Há mais de 30 anos foi proposto um modelo para explicar como o sistema nervoso central promove a regulação do sistema cardiovascular, onde os núcleos vasomotores do bulbo seriam as principais estruturas envolvidas no controle do reflexo cardiovascular. Segundo este modelo, o núcleo do trato solitário (NTS) é o primeiro núcleo a integrar as informações cardiovasculares vindas dos baroceptores e também parece integrar vias descendentes provenientes de núcleos superiores como o hipotálamo, importantes para as reações de alerta e defesa. Do NTS saem projeções excitatórias para a região caudoventrolateral (CVL) do bulbo, a qual inibe a região rostroventrolateral (RVL). Esta última região constitui a principal fonte de eferências excitatórias para os neurônios simpáticos pré-ganglionares (SPN), sendo responsável pelo tonus simpático para o coração e vasos. Projeções importantes do CVL para estruturas diencefálicas (núcleo preóptico mediano, núcleo paraventricular do hipotálamo e núcleo supraóptico) também estão envolvidas no controle da composição e/ou volume do compartimento extracelular. A área depressora gigantocelular (GiDA) constitui outro possível centro vasomotor envolvido nos ajustes de fluxo sangüíneo por meio de projeções diretas para o SPN. No entanto, o meio pelo qual a GiDA exerce seu efeito vasodepressor ainda é desconhecido.

Nos últimos 10 anos, nosso laboratório tem se dedicado a deslindar as vias e mecanismos neurais associados à regulação do fluxo sangüíneo visceral e muscular. Resultados obtidos ao longo destes estudos resultaram em evidências que são incompatíveis com o modelo proposto.

Descritores: Pressão Arterial. NTS. RVL. CVL.

\section{1- INTRODUÇÃO}

A perfusão do leito vascular capilar constitui um elemento essencial da homeostasia. É através do fluxo sangüíneo que percorre este território e das tro- cas que aí se realizam, que o interstício pode ser renovado, mantendo-se nas condições ideais para a homeostasia celular. A perfusão do leito capilar depende de níveis adequados de pressão sangüínea arterial, uma vez que esta constitui a força motriz que 
propulsiona o sangue até o território capilar. Há mais de um século, sabe-se que o tronco cerebral e, mais especificamente, o bulbo (ou medula oblongata) constitui o segmento crítico para a regulação neural da pressão arterial. No entanto, foi só a partir da década de 70, que um avanço significativo nesta área ocorreu. Em uma série de trabalhos basilares, Guertzenstein e colaboradores ${ }^{1 / 4}$ demonstraram que regiões restritas do tegmento ventrolateral do bulbo continham neurônios cuja atividade era fundamental para a manutenção do tono vasomotor e da pressão arterial. Literalmente, milhares de artigos científicos publicados desde então, confirmaram e expandiram conceitos firma- dos pelas observações originais de Guertzenstein e colaboradores, o que é a maior prova da qualidade excepcional destes estudos.

A Figura 1 apresenta uma representação esquemática dos principais núcleos vasomotores do bulbo e as suas relações com outras áreas do sistema nervoso central envolvidas na regulação cardiovascular. De acordo com este modelo:

1) o tono simpático para o coração, vasos de resistência e medula adrenal se origina de neurônios simpáticos pré-ganglionares (SPN) localizados na coluna intermediolateral (IML) nos segmentos torácicos da medula espinal;
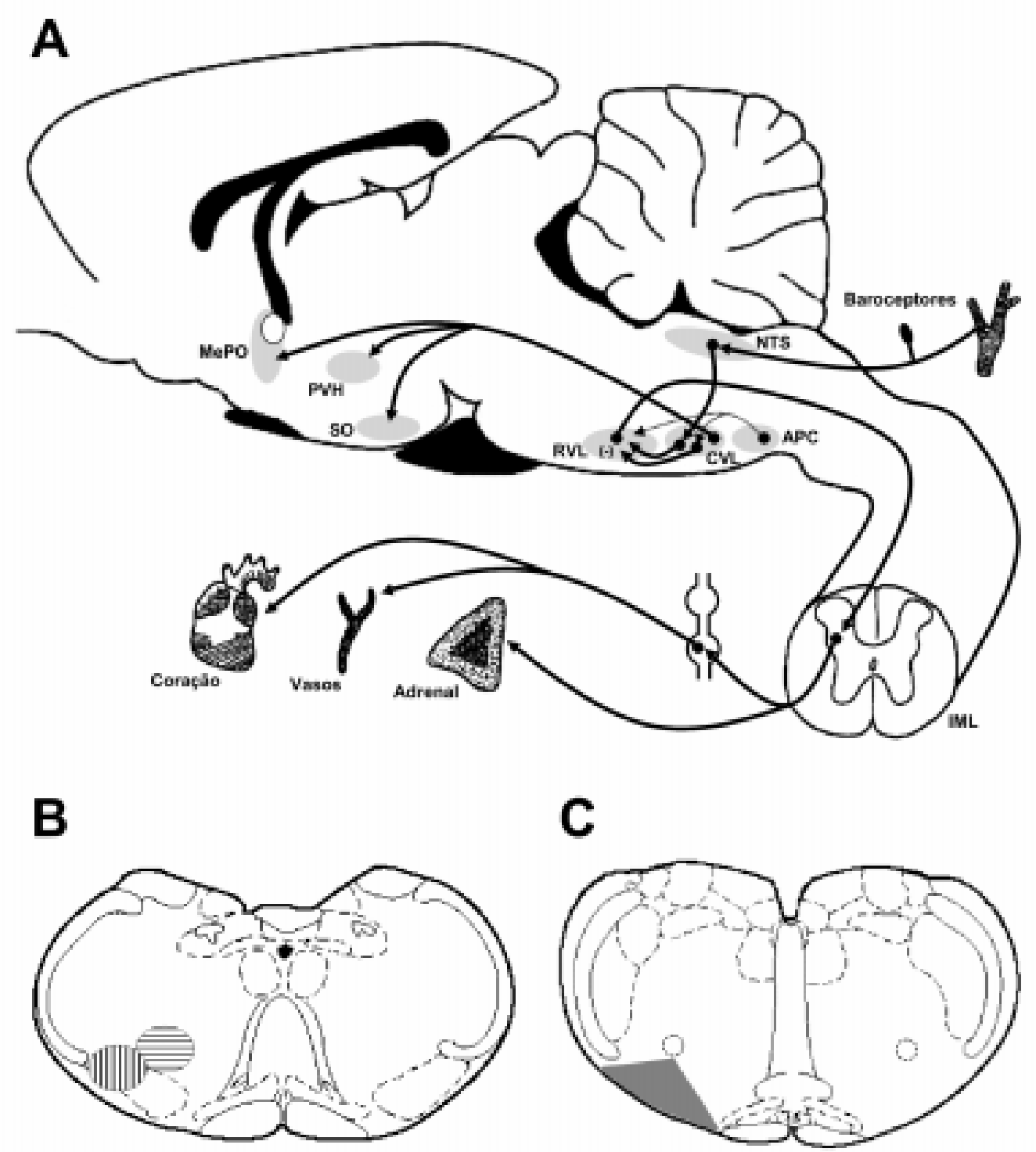

Figura 1: Representação esquemática de um corte sagital do encéfalo com os principais núcleos vasomotores presentes no tegmento ventrolateral do bulbo e suas conexões com outros núcleos autonômicos do sistema nervoso central. 
2) os SPN são tonicamente excitados por projeções diretas de um grupo restrito de neurônios localizados na porção rostroventrolateral do bulbo (RVL);

3) os neurônios do RVL são controlados por projeções inibitórias (tônicas e reflexas) de neurônios situados na porção caudoventrolateral do bulbo (CVL);

4) os reflexos baroceptores utilizam a via inibitória CVL-RVL para modulação da atividade simpática do coração, vasos e medula adrenal: a estimulação dos baroceptores arteriais excita neurônios de projeção no núcleo do trato solitário (NTS), que excitam neurônios no CVL, aumentando a sua inibição sobre os neurônios do RVL, o que resulta em diminuição do tono simpático para o coração e vasos;

5) além de suas projeções inibitórias ao RVL, o CVL contém neurônios distintos que se projetam para núcleos diencefálicos como o núcleo paraventricular do hipotálamo (PVH) e núcleo supra-óptico, modulando a secreção de vasopressina;

6) neurônios localizados na porção mais caudal do tegmento bulbar constituem a área pressora caudal (CPA). Essa região contribue para a geração do tono vasomotor e manutenção da pressão arterial, entretanto as vias pelas quais a CPA exerce o seu papel ainda não são conhecidas.

Trata-se, é claro, de uma descrição sumária, que visa apenas a delinear as principais conclusões derivadas destes 30 anos de pesquisas desde os trabalhos pioneiros de Guertzenstein e colaboradores (para uma revisão detalhada destes conceitos ver Colombari et al., 2001 ${ }^{5}$.

Nos últimos 10 anos, nosso laboratório tem se dedicado a deslindar as vias e mecanismos neurais associados à regulação do fluxo sangüíneo visceral e muscular. Resultados obtidos ao longo destes estudos resultaram em evidências que são incompatíveis com o modelo proposto. Neste artigo pretendemos descrever brevemente estes resultados, indicando as sugestões que eles contém e indicando os pontos em que eles discordam de nossa visão atual.

\section{2- A VASODILATAÇÃO MUSCULAR DAS REAÇÕES DE ALERTA E A REGULAÇÃO CARDIOVASCULAR}

As reações de alerta e defesa constituem uma ampla classe de comportamentos exibidos por numerosas espécies, inclusive a humana. Embora quase que exclusivamente referidos como os comportamentos exibidos por um indivíduo frente a estímulos potenci- almente lesivos ou um agressor mais poderoso, os comportamentos defensivos provavelmente constituem um contínuo, incluindo desde o simples abanar de uma orelha ou cauda para afastar um inseto, a retração de um membro (comportamento de flexão) ou a cabeça de um agente nocivo, a esquiva ou fuga, manifestações verbais de desculpa, de justificativas, etc. ${ }^{6}$

Os comportamentos de alerta e defesa englobam diversos ajustes motores e neurovegetativos com a intenção de preparar e dar suporte metabólico para situações de luta ou fuga ${ }^{7,8}$. Os ajustes cardiovasculares incluem: hipertensão, taquicardia, vasoconstrição visceral e vasodilatação muscular. Uma vez que estes comportamentos geralmente se acompanham de intensa ativação motora, a vasodilatação muscular tem sido admitida como um de seus componentes principais ao fornecer suporte metabólico.

Embora só possam ser observadas plenamente em animais despertos e expostos a estímulos naturais, os ajustes vegetativos das reações de defesa podem ser provocados em animais anestesiados pela estimulação elétrica ou química do sistema nervoso central.

A estimulação elétrica de regiões hipotalâmicas ao redor do fornix, por exemplo, desencadeia reações caracterizadas por um aumento transitório da pressão arterial, taquicardia, vasoconstrição visceral e intensa vasodilatação muscular esquelética ${ }^{7 / 10}$, por esta razão esta área tem sido denominada área de defesa hipotalâmica (HDA).

Utilizando bloqueios farmacológicos seletivos, em trabalhos anteriores de nosso laboratório ${ }^{9,10}$, demonstramos que a vasodilatação muscular provocada por estimulação da HDA em ratos anestesiados parece resultar de três mecanismos distintos: 1) redução do tônus vasoconstrictor simpático, 2) liberação de catecolaminas pelas células cromafins localizadas na medula da adrenal e 3) ativação de um sistema envolvendo a liberação de óxido nítrico e fatores nitrosila$\operatorname{dos}^{11}$. Em um estudo posterior, demonstramos que o bloqueio de sinapses glutamatérgicas no RVL é capaz de bloquear completamente esta vasodilatação9. Em conjunto, estes resultados indicam que a vasodilatação muscular das reações de alerta parece depender da excitação glutamatérgica de neurônios no RVL e, a partir daí, ativação dos três mecanismos efetores já citados.

Em uma continuidade a estes estudos buscamos identificar as possíveis vias e estruturas envolvidas na ativação do RVL durante as reações de alerta ${ }^{9}$. Estudos anteriores demonstram que além de suas 
já bem conhecidas ações nas vias reflexas dos baro e quimioceptores, o NTS parece participar como sítio de integração de respostas cardiovasculares durante as reações de alerta e defesa ${ }^{12,13}$. A estimulação química do NTS provoca vasodilatação muscular que parece envolver a redução do tono vasoconstrictor simpático, a liberação de catecolaminas pela adrenal e a liberação de NO de acordo com o subtipo de receptor purinérgico estimulado ${ }^{14,15}$. O NTS apresenta projeções diretas para o RVL, cujo significado funcional permanece ainda em grande parte desconhecido. No NTS, o ATP e a adenosina derivada do catabolismo extracelular atuam como importantes neurotransmissores / neuromoduladores na regulação do fluxo sangüíneo regional muscular e visceral ${ }^{13,16,17}$. Estudos autoradiográficos $^{18 / 21}$ demonstram alta densidade de receptores P1 (adenosina) e P2 (ATP) nos centros cardiovasculares e respiratórios do NTS. Estes estudos anteriores demonstraram que a adenosina é rapidamente liberada no NTS durante a estimulação elétrica da $\mathrm{HDA}^{12}$, participando na ativação dos subtipos de receptores $\mathrm{A} 1^{19,22}$. Estes estudos nos levaram a investigar a hipótese de que as reações de alerta possam envolver a ativação de uma via HDA-NTS-RVL e que a neurotransmissão purinérgica do ATP possa participar destas reações.

Como podemos observar na Figura 2, em ratos anestesiados, a microinjeção no NTS de agonistas purinérgicos do subtipo P2x á,âmeATP (100pmol/50nl) desencadeou hipotensão, bradicardia e vasodilatação
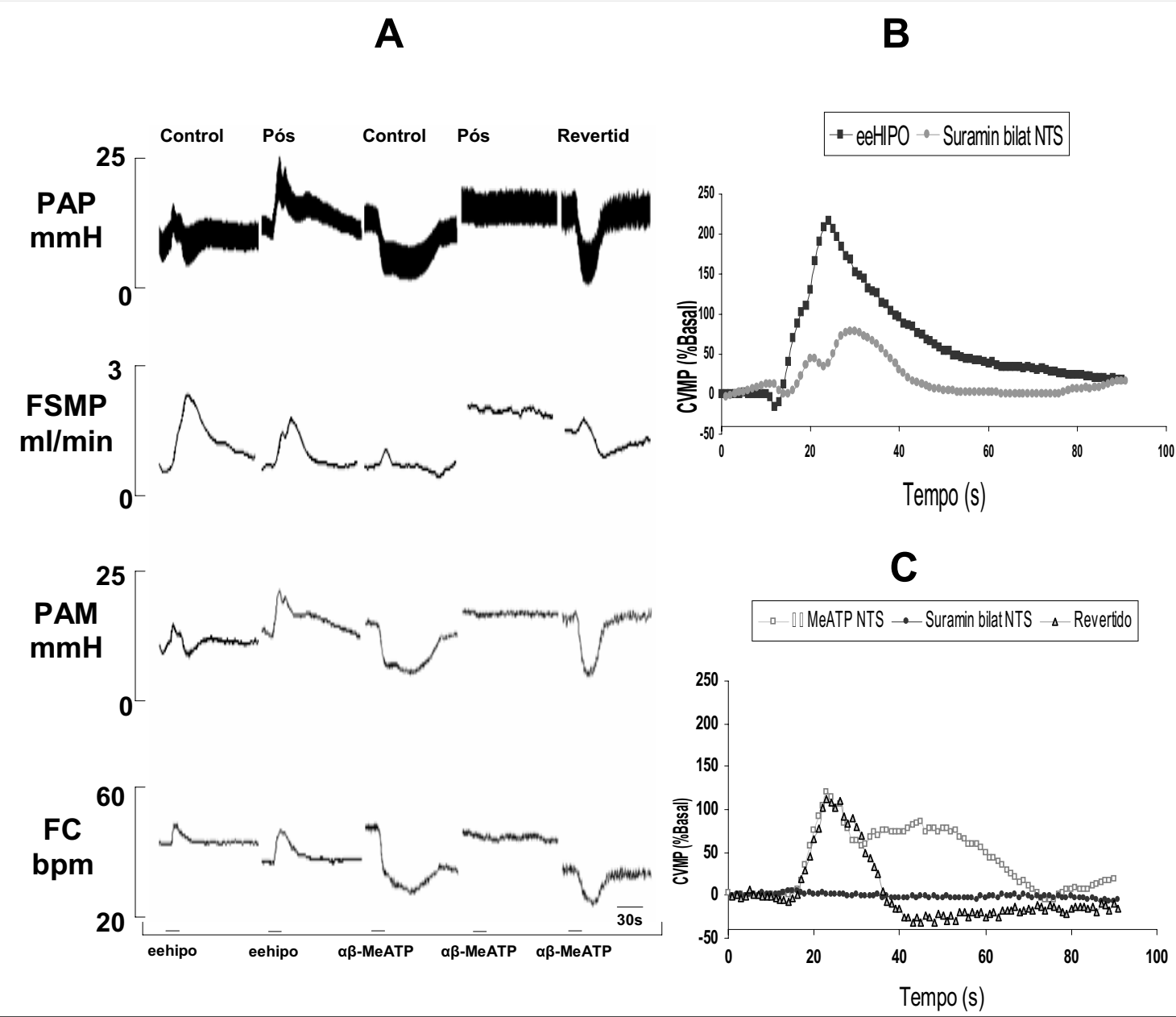

Figura 02: (A) Efeitos da estimulação elétrica do hipotálamo (eehipo) e da microinjeção de ba-meATP (100pmol/50nl) no NTS intermédio na pressão arterial pulsátil (PAP), pressão arterial média (PAM), freqüência cardíaca (FC) e fluxo sangüíneo para os membros posteriores (FSMP) antes e após o bloqueio bilateral no NTS com Suramin (100pmol/50nl) em ratos anestesiados. (B) Curvas de condutância vascular dos membros posteriores antes (quadrados) e após (círculos) o bloqueio bilateral com Suramin no NTS, durante a estimulação elétrica do hipotálamo expresso como \% do valor basal. (C) Curvas de condutância vascular dos membros posteriores antes (quadrados), após (círculos) e após a reversão do bloqueio (triângulos) bilateral com Suramin no NTS, durante a microinjeção de ba-meATP no NTS expresso como \% do valor basal 
nos membros posteriores, indicando que a neurotransmissão purinérgica é capaz de promover vasodilatação muscular. Nestes animais, a estimulação da HDA provoca hipertensão, bradicardia e vasodilatação muscular. A microinjeção bilateral de suramin (100pmol/ 50nl), um antagonista P2x, no NTS é capaz de reduzir drasticamente a vasodilatação muscular de membros posteriores, sem afetar as respostas pressoras ou taquicárdicas induzidas pela estimulação elétrica da HDA. Esta dose de suramin é capaz de bloquear completamente as respostas cardiovasculares provocadas pela microinjeção de seu agonista á,âmeATP., indicando que a dose de suramin utilizada é eficiente no bloqueio destes receptores. O bloqueio do suramim é reversível, i.e., após 60 a $80 \mathrm{~min}$, a vasodilatação muscular em resposta à estimulação da HDA e à microinjeção do agonista são restabelecidas. Os níveis basais de pressão arterial, freqüência cardíaca e fluxo sangüíneo para os membros posteriores não são modificados pelo bloqueio bilateral dos receptores $\mathrm{P} 2 \mathrm{x}$. Estes resultados indicam que a redução da vasodilatação pelo suramim no NTS não é um efeito lesivo ou inespecífico ou devido a modificações nos níveis de perfusão dos tecidos.

Para determinarmos a relação entre a ativação dos receptores $\mathrm{P} 2 \mathrm{x}$ localizados nos neurônios do NTS com o núcleo RVL durante os ajustes hemodinâmicos, microinjetamos $\alpha, \beta$ meATP (100pmol/50nl) no NTS. Em seguida, realizamos o bloqueio bilateral de receptores glutamatérgicos no RVL através da microinjeção bilateral de ácido quinurênico (5nmol/50nl). Passados vinte minutos microinjetamos novamente $\alpha, \beta$ meATP no NTS. A vasodilatação dos membros posteriores foi totalmente abolida, assim como a hipotensão e a bradicardia. Passados 90 minutos de bloqueio, todos os ajustes retornam às suas condições normais, demonstrando uma reversão desse bloqueio. Os valores basais de pressão arterial, freqüência cardíaca e fluxo sangüíneo para as patas posteriores permaneceram inalterados após o bloqueio no RVL.

Em conjunto, estes resultados são compatíveis com a hipótese de que às respostas de alerta provocadas por estimulação hipotalâmica envolvem a ativação de uma via purinérgica no NTS, e a partir da ativação desse núcleo, o RVL é ativado por vias glutamatérgicas, mobilizando vias neurais e mecanismos capazes de promover intensa vasodilatação muscular.

\section{3- A ÁREA GIGANTOCELULAR DEPRES- SORA (GIDA) E A GERAÇÃO DO TONO VASOMOTOR SIMPÁTICO}

Em um estudo de 1994, Aicher et al. ${ }^{23}$ demonstraram que a estimulação química por microinjeções de glutamato em uma área localizada na região ventral e medial do bulbo provocava hipotensão e redução da atividade simpática, caracterizando esta região como uma região vasodepressora. Por esta razão, esta região foi então denominada de Área Depressora Gigantocelular (GiDA)*1. Mais significante, todavia, era a observação de que a microinjeção do agente neuroexcitatório/neurotóxico ácido caínico nesta região provocava hipertensão arterial acentuada de origem neurogênica, ou seja, com aumento da atividade simpática. Estes resultados sugeriam que a GiDA se constituía em uma região com atividade simpatoinibitória tônica, i.e., a atividade dos neurônios ali presentes era fundamental para inibir a geração do tono vasomotor. Estes resultados não eram compatíveis com o modelo de geração do tono simpático até então adotado e descrito anteriormente neste trabalho. Ainda utilizando técnicas de microinjeção de aminoácidos excitatórios e analisando os efeitos reflexos e tônicos sobre a atividade simpática e a pressão arterial, Aicher e Reis, $1997^{24}$ demonstraram que a GiDA era funcionalmente distinta de outras regiões do tegmento medular cujo envolvimento com a regulação cardiovascular já era bem conhecido como, por exemplo, o CVL ou os núcleos da rafe.

Combinando técnicas de rastreamento neuronal retrógrado e anterógrado e microscopia eletrônica, estes autores buscaram estabelecer as possíveis vias pelas quais a GiDA exercia os seus efeitos simpatoinibitórios. Os resultados obtidos demonstraram que a GiDA mantém conexões com outros núcleos autonômicos da medula oblonga como a região ventral do núcleo reticular ventral, os núcleos reticulares parvocelular, pálido e obscuro e o núcleo do trato solitário (subnúcleos comissural e medial), além destes locais, algumas poucas fibras marcadas foram observadas no RVL. Todavia, as suas principais eferências parecem se dirigir para a medula espinal e, mais especificamente, para os segmentos torácicos da medula espinal, na região da coluna intermediolateral. Combinando a marcação anterógrada de fibras provenien-

\footnotetext{
* De maneira a evitar a proliferação de abreviaturas e sinônimos desnecessários, nesta revisão optamos por adotar a abreviação GiDA, originalmente proposta pelos autores.
} 
tes da GiDA com a identificação retrógrada de neurônios simpáticos pré-ganglionares, Aicher et al. $1995^{25}$ demonstraram que a GiDA mantém conexões sinápticas diretas inibitórias com neurônios simpáticos préganglionares que inervam células cromafins da medula da adrenal. No entanto, essas evidências anatômicas não suportam, de modo inequívoco, a função vasodepressora/simpatoinibitória da GiDA. Estes achados anatômicos eram compatíveis com os achados funcionais que indicavam um papel inibitório da GiDA sobre a geração da atividade simpática. Todavia, à inibição destes neurônios, provocaria apenas redução da secreção de catecolaminas circulantes, um efeito que não poderia explicar completamente a significativa hipotensão resultante da estimulação da GiDA.

Outros resultados funcionais sobre a atividade tônica da GiDA permanecem em grande parte desconhecidos. É intrigante a observação, por exemplo, que microinjeções unilaterais de muscimol (agonista de receptores GABAérgico do subtipo A) provoca hipotensão, ao passo que microinjeções bilaterais provocam hipertensão ${ }^{26}$. Outra evidência interessante é que microinjeções de clonidina (agonista de adrenoceptores do subtipo á 2 na GiDA produz bradicardia e hipotensão que persiste por até 2 horas, sendo estes efeitos similares aos da clonidina no RVL ${ }^{27}$. Vários estudos têm demonstrado que o efeito antihipertensivo da clonidina e outros adrenoceptores á 2 são mediados principalmente ou somente por meio de regiões medulares específicas, incluindo o NTS, a formação reticular gigantocelular, e particularmente o $\mathrm{RVL}^{28,29,30}$. No entanto, os resultados demonstrados por Aicher e Drake, $1999^{27}$, indicam que a clonidina possui o mesmo efeito em duas regiões funcionalmente distintas do bulbo: a GiDA, que é uma região simpatoinibitória e vasodepressora tonicamente ativa, e o RVL, que é uma região simpatoexcitatória e vasopressora tonicamente ativa ${ }^{31,32}$. A partir destas evidências, temos desenvolvido uma linha de pesquisa que visa colaborar com a caracterização anatômica e funcional desta possível nova região bulbar envolvida na regulação cardiovascular.

Dentre os estudos anatômicos realizados em nosso laboratório, focalizamos, com mais atenção, a distribuição das eferências da GiDA nos núcleos autonômicos da região torácica da medula espinal. Para isso, foram realizados depósitos iontoforéticos do traçador anterógrado BDA na GiDA. Após o tempo de sobrevida necessário para o transporte do traçador até a medula espinal, os animais foram sacrificados e os cortes da região torácica da medula espinal foram submetidos à imunoistoquímica para acetilcolina transferase (ChAT).

Os neurônios simpáticos pré-ganglionares são os últimos neurônios das vias do sistema nervoso central envolvidos com a regulação da função autonômica. Todos estes neurônios são colinérgicos e, portanto, contêm ChAT, sendo assim, a imunoistoquímica para esta enzima serve como marcador para estes neurônios.

Neste estudo, observamos que as projeções da GiDA descendem pelo funículo lateral ipsolateral exclusivamente. A maioria dessas fibras descendentes se projeta e arboriza-se na região da IML. No entanto, um número considerável de fibras cruza a linha média após entrar na substância cinzenta e arborizase por todo o seu trajeto.

Como conseqüência desta intensa arborização observa-se botões terminais não só na IML ipsolateral, como também na IML contralateral, e em outros núcleos autonômicos da medula, como a área autonômica central e o núcleo intercalado. Nos núcleos autonômicos citados acima podemos observar aposições entre as eferências da GiDA e os neurônios simpáticos marcados com ChAT.

A possibilidade das eferências da GiDA contatarem um grande número de SPNs ao longo de todo o eixo rostro caudal da região torácica da medula espinal, além dos SPNs que originam a inervação simpática da adrenal, juntamente com o efeito fisiológico demonstrado por Aicher et al., 199423, nos indica que esta área bulbar provavelmente exerce uma influência mais ampla e funcionalmente distinta na regulação autonômica do que a sabida até o momento.

A partir das observações citadas acima, procuramos outras evidências anatômicas que justifiquem o efeito simpatoinibitório da GiDA. A existência de conexões da GiDA com SPNs que originam a inervação a outros alvos simpáticos como o coração e a vasculatura visceral e muscular tem sido objeto de nosso trabalho.

Inicialmente foram feitos depósitos de Amino Dextrana Biotinilada (BDA) na GiDA (Figura 3c) e microinjeções de Fluorogold no gânglio celíaco. Na análise da região torácica da medula espinal foram observadas aposições na IML (Figura 3b). Frequentemente, foram visualizados fibras e neurônios marcados no núcleo intercalado, no entanto sem aposições.

Estas aposições ocorrem em ramificações proximais, indicando que a GiDA exerce um forte efeito inibitório sobres estes neurônios, já que, sinapses em locais proximais dos neurônios geralmente causam uma inibição absoluta do sinal ${ }^{33}$. 
Este conjunto de informações nos permite supor que a GiDA inerva SPNs que dão origem a inervação simpática dos vasos mesentéricos e levando em consideração a função desta região e os resultados obtidos, é razoável supor que essas sinapses são provavelmente inibitórias. No entanto, a natureza química e fisiológica destas sinapses ainda precisam ser demonstradas, assim como, a conexão da GiDA com neurônios simpáticos pré-ganglionares que originam a inervação simpática para outros territórios.

As eferências da GiDA provavelmente contêm os neurotransmissores que já foram localizados na formação reticular gigantocelular, como $\mathrm{GABA}^{34}$, glicina $^{34,35}$ e serotonina ${ }^{36}$. Destes neurotransmissores, o candidato mais provável é o GABA, já que neurônios reticulo-espinais da área gigantocelular contêm $\mathrm{GABA}^{37}$ e SPNs recebem contatos sinápticos GABAérgicos $^{38}$.

Dentre os primeiros estudos funcionais da GiDA realizados em nosso laboratório buscamos caracterizar os efeitos da estimulação química da GiDA sobre a condutância vascular nos territórios muscular e visceral, bem como investigar os seus principais mecanismos envolvidos. Neste estudo, pode-se observar que microinjeções de L-glutamato na GiDA produz redução da pressão arterial média (PAM) e FC; aumentos no fluxo sangüíneo de membros posteriores e fluxo sangüíneo renal (FSR), além de vasodilatação dos territórios vasculares do trem posterior e renal. Após a administração de prazosin (antagonista de adrenoceptores $\mathrm{a}_{2}$ ) observamos que as respostas de PAM e FC e a vasodilatação nos territórios vasculares do trem posterior e renal induzidas por microinjeções de L-glutamato na GiDA foram significantemente reduzidas. Em conjunto, estes resultados sugerem que a hipotensão, bradicardia e vasodilatação visceral e muscular por estimulação da GiDA são decorrentes da redução do tono simpático vasoconstrictor.

Em outros experimentos, analisamos o papel do RVL nas respostas cardiovasculares induzidas por inativação da GiDA com ácido caínico (KA). Nestes estudos, observamos que microinjeções bilaterais de KA na GiDA produziram acentuada hipertensão arterial com hiperatividade simpática. Nesta condição, a inativação química do RVL com muscimol não reverte a hiperatividade simpática induzida por KA na GiDA, embora os valores de pressão arterial apresentem-se reduzidos. Além disso, observamos em outro grupo de animais que se a inativação química do RVL com muscimol precede as microinjeções bilaterais de KA na GiDA, os efeitos hipertensores e de hiperatividade simpática induzidos por KA ainda assim ocorrem (Figura 3a). Estes resultados sugerem que os efeitos da GiDA sobre o sistema cardiovascular independem da integridade funcional dos neurônios do RVL ${ }^{39}$.

\section{4- PAPEL DOS NEURONIOS A1 NA REGULA- ÇÃO DO VOLUME EXTRACELULAR}

Imediatamente caudal ao RVL situa-se a região do CVL. Embora a descrição original dessa região e suas principais características tenha sido feita por Guertzenstein e colaboradores simultaneamente à descrição do RVL, é surpreendente como o desenvolvimento dos conhecimentos sobre o CVL tem se desenvolvido tão lentamente quando comparado ao RVL. Talvez uma das principais causas desta discrepância deva-se ao fato que, ao contrário do RVL, o CVL parece ser uma área funcionalmente heterogênea, contendo diversos sistemas anatômica e funcionalmente muito distintos (é por esta razão que no caso do CVL é preferível utilizar o termo "região CVL" e não "núcleo CVL") .

O CVL parece conter pelo menos dois circuitos que podem ser distintos quanto a sua natureza: um envolvido com a regulação do tono vasomotor simpático e outro que parece envolvido com a regulação do volume extracelular. Como descrito na figura 01, neurônios do CVL projetam-se para o RVL, onde exercem modulação inibitória (provavelmente GABAérgica) sobre os neurônios do RVL que se projetam para a IML.

Estudos neuroanatômicos indicam que, além de suas projeções para o RVL, neurônios do CVL projetam-se para áreas hipotalâmicas como o núcleo paraventricular do hipotálamo (PVH) e núcleo supraóptico (SON). Estes núcleos são particularmente importantes por conterem neurônios magnocelulares responsáveis pela síntese, transporte e liberação de vasopressina por terminais axonais na neurohipófise $e^{40,41,42}$. A liberação de vasopressina constitui um dos vários mecanismos que contribuem para manter a homestase cardiovascular e hidromineral, sendo importante mecanismo para a reabsorção de água e aumento da resistência vascular periférica.

O CVL também envia densa projeção para a região anteroventral do terceiro ventrículo (AV3V), tendo particular importância o núcleo pré óptico mediano (MePO $)^{40,41,42}$. Este alvo é um elemento central na regulação do equilíbrio hidroeletrolítico. 


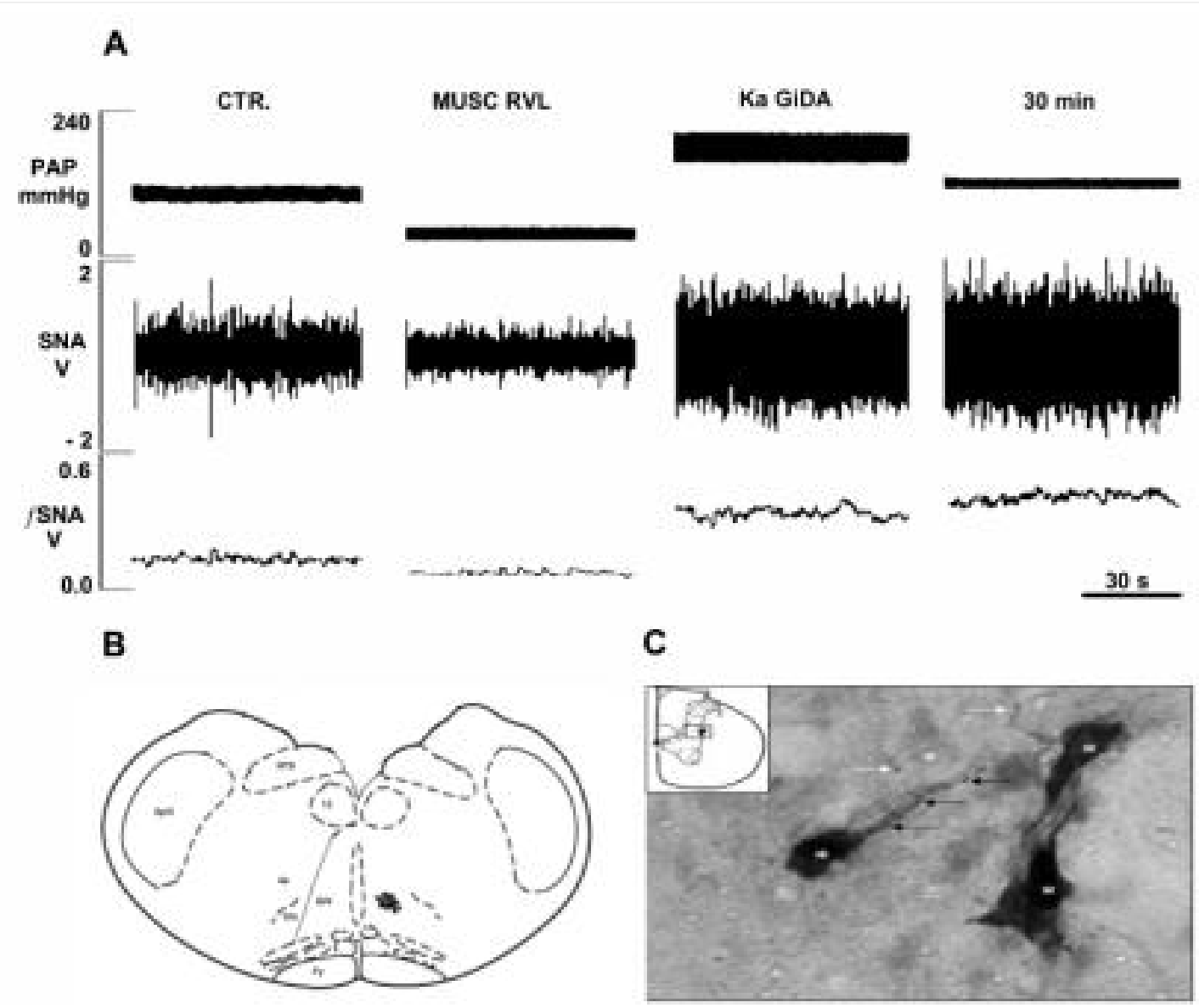

Figura 3: (A) Registro da pressão arterial pulsátil (PAP), atividade simpática do nervo esplâncnico (SNA) e integral da atividade simpática ('SNA) em ratos anestesiados durante o período controle (CTR), após inativação bilateral do RVL com muscimol (MUSC RVL), 5 (ka GiDA) e 30 minutos após microinjeções bilaterais de ácido caínico ( $1 \mathrm{nmol} / 50 \mathrm{~nL})$ na GiDA. (B) desenho semiesquemático de um corte coronal do bulbo mostrando o local de depósito de BDA na GiDA (hachurado- lado direito da figura). No lado esquerdo pode-se identificar as seguintes estruturas anatômicas: NTS (núcleo do trato solitário); Sp5I (núcleo espinal do nervo trigêmeo); 12 (núcleo hipoglossal); Gi (núcleo reticular gigantocelular); GiV (núcleo reticular gigantocelular ventral); 12n (raiz do nervo hipoglosso) e Py ( trato piramidal). (C) Fotomicrografia de campo claro de corte transversal e desenho esquemático da região torácica da medula espinal. No desenho esquemático nota-se a localização da IML em negro dentro do detalhe. Na fotomicrografia pode-se observar SPNs que dão origem a inervação do gânglio celíaco marcados retrogradamente com Fluorogold (asteriscos brancos). Nestes neurônios podemos observar locais de possíveis aposições (setas pretas) entre fibras marcadas com BDA (setas brancas) e os SPN marcado com Fluorogold.

Estas eferências do CVL dirigidas a diversos alvos diencefálicos representam as vias envolvidas na regulação do volume extracelular. As vias ascendentes do CVL originam-se predominantemente de neurônios noradrenérgicos dos grupamentos A1 localizados nesta região ${ }^{40,41,42}$. É importante ressaltar que os neurônios A1 não se projetam ao RVL e, portanto, não estão primordialmente envolvidos na regulação do tono simpático.

Sabe-se que as informações viscerais provenientes de receptores periféricos (como barorreceptores, receptores cardiopulmonares, quimiorreceptores e osmorreceptores periféricos localizados na veia por- ta) enviam projeções para o NTS. A partir deste, as informações podem alcançar diferentes destinos no sistema nervoso central, incluindo-se o tegmento ventrolateral do bulbo (RVL e CVL).

Nos últimos anos, trabalhos de nosso laboratório $^{43 / 46}$ têm se dedicado a estudar as aferências, áreas de integração bulbar e eferências das respostas hemodinâmicas provocadas por alterações agudas do volume e concentração do fluido extracelular. Entre os diversos modelos experimentais empregados, temos utilizado a infusão de pequenos volumes $(0,5 \mathrm{ml})$ de salina hipertônica ( $\mathrm{NaCl} 3 \mathrm{M})$.

Em estudos anteriores demonstramos que, em 
ratos anestesiados, a infusão de $\mathrm{NaCl} 3 \mathrm{M}$ provoca hipertensão transitória e aumentos mantidos do FSR e da condutância vascular renal $(\mathrm{CVR})^{43 / 46}$. Admite-se que o aumento da CVR, i.e., a vasodilatação do território renal, constitua um dos mecanismos essenciais para promover a excreção da carga de sódio infundida e o restabelecimento das condições normais de volemia e tonicidade. Os resultados obtidos indicam que a denervação sinoaórtica abole a vasodilatação renal induzida pela infusão de salina hipertônica, enquanto que a vagotomia bilateral (bloqueando as aferências de receptores cardiopulmonares) não modifica essa resposta ${ }^{46}$. Estes dados indicam que são os barorreceptores arteriais, e não os receptores cardiopulmonares, responsáveis pela origem da vasodilatação renal. Além disso, a vasodilatação renal pode também ser observada após desnervação renal, o que indica que ela pode ser devida a fatores humorais. Dentre os possíveis peptídeos vasoativos envolvidos com esta resposta, o papel do petídeo natriurético atrial (ANP) tem sido investigado. O ANP é secretado pela infusão de salina hipertônica, e sua secreção é modulada por aferentes dos baroceptores ${ }^{47}$.

A partir destes resultados buscamos testar a hipótese que a infusão de salina hipertônica provoca a ativação de aferentes dos baroceptores que, através da via NTS-CVL, ativariam alvos diencefálicos como o MePO (núcleo integrante da região $\mathrm{AV} 3 \mathrm{~V}$ ), ativando os mecanismos que resultariam em vasodilatação renal.

Como mencionado anteriormente, a injeção endovenosa de salina hipertônica promove aumento transitório da pressão arterial e aumentos mantidos de FSR e da CVR. Entretanto, ao realizarmos lesão eletrolítica da região $\mathrm{AV} 3 \mathrm{~V}$, os aumentos de pressão, fluxo e condutância são abolidos ${ }^{45}$. Estes resultados evidenciam que a região $\mathrm{AV} 3 \mathrm{~V}$ participa na integração das respostas cardiovasculares à salina hipertônica (Figura 4a).

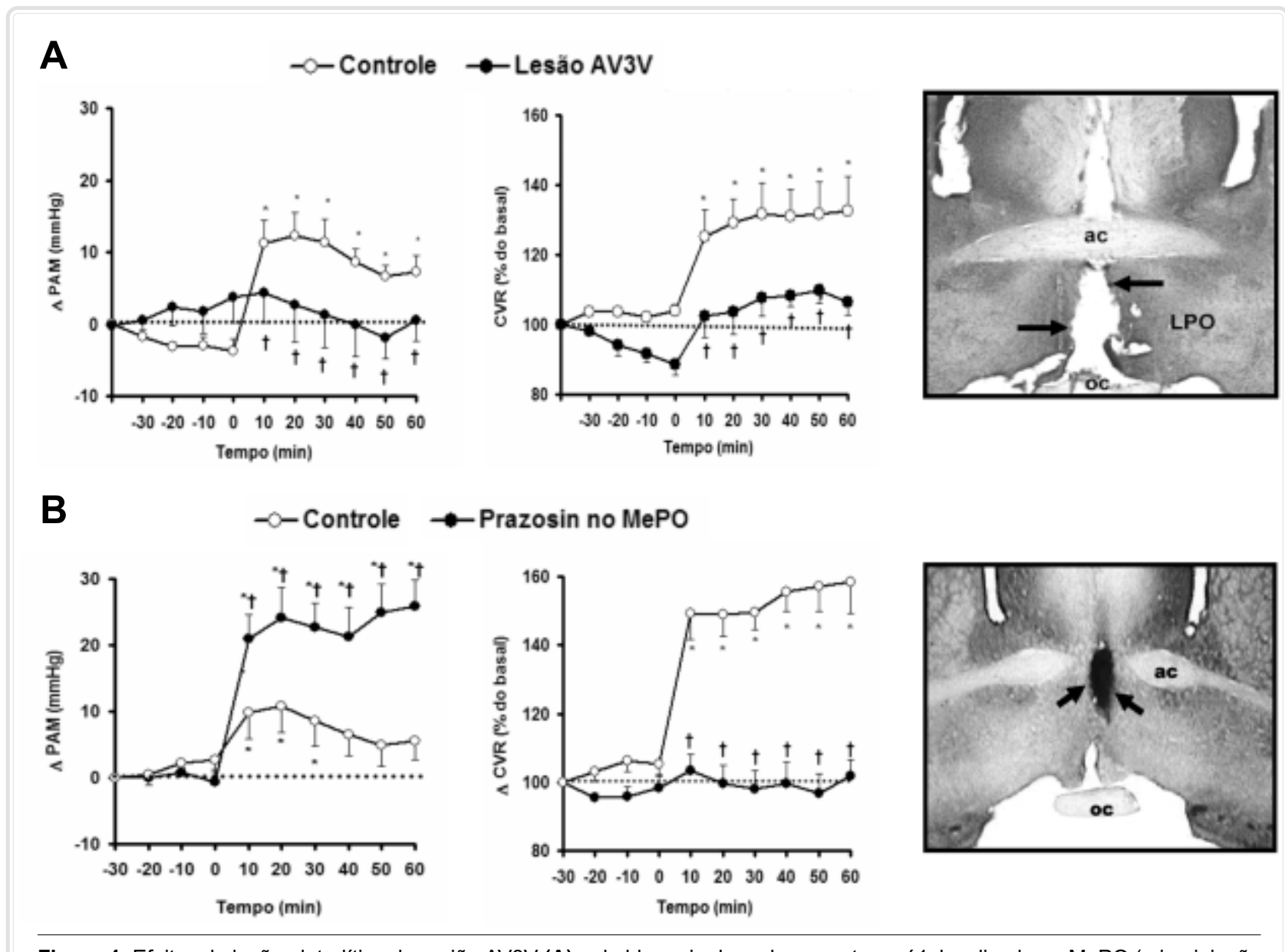

Figura 4: Efeitos da lesão eletrolítica da região AV3V (A) e do bloqueio dos adrenoceptores á1 localizado no MePO (microinjeção do antagonista á1 prazosin; B) sobre as variações da pressão arterial média (PAM) e da condutância vascular renal (CVR) induzidas pela sobrecarga de sódio $(\mathrm{NaCl} 3 \mathrm{M})$ em ratos anestesiados. 
Estudos neuroanatômicos e imunoistoquímicos demonstram que a região $\mathrm{AV} 3 \mathrm{~V}$ recebe importantes projeções noradrenérgicas do CVL. Desta forma investigamos o papel dos receptores adrenérgicos do subtipo a1 nas respostas cardiovasculares induzidas após a sobrecarga de sódio. Os resultados obtidos demonstram que a microinjeção prévia de prazosin (antagonista a1) reduz o FSR e abole a vasodilatação renal observada após salina hipertônica (Figura 4b). Se a microinjeção de prazosin é realizada mesmo após o pleno desenvolvimento da vasodilatação, ou seja, após uma hora da injeção endovenosa de salina hipertônica, ainda assim esta droga é capaz de bloquear a vasodilatação renal. Estes resultados em conjunto, são indi- cativos que os receptores a 1 presentes no MePO participam na gênese e manutenção da vasodilatação renal provavelmente através de modulação da atividade simpática e liberação de ANP. Portanto, com base nos subtratos neuroatanômicos anteriormente citados, podemos supor que essas projeções noradrenérgica do grupamento A1 para os núcleos MePO, estimulada por aferências baroceptoras, podem modular as respostas cardiovasculares, endócrinas e neurais induzidas por variações na composição e/ou volume do compartimento extracelular. O desenvolvimento atual deste projeto envolve lesões seletivas do grupamento A1 de modo a confirmar estas hipóteses.

Cravo SL, Rosa DA, Kalassa F, Korim WS, Hinrichs JM, Ferreira-Neto ML, Di Mônaco LR, Pedrino GR. Ventrolateral medulla and the cardiovascular control: new evidence new doubts. Medicina (Ribeirão Preto) 2006; 39 (1): 89-100.

ABSTRACT : More than 30 years ago a model was proposed trying to explain how the central nervous system controls the cardiovascular adjustments. According to this model the medullary vasomotor nuclei are the main structures involved in the cardiovascular reflex control. It also shows the nucleus tractus solitarius (NTS) as being the first integrative center for the baroceptors afferents and the descending pathways from supramedullary nuclei of hypothalamus, which contribute at defense and alert reactions. The NTS exhibits excitatory projections to the caudal ventral lateral medulla (CVL) which inhibits the rostral ventral lateral medulla (RVL). The RVL is the main source of excitatory inputs to the sympathetic preganglionic neurons, being responsible for the sympathetic tonus to heart and blood vessels. Important projections from CVL to diencephalon structures (Median preoptic nucleus, Hypothalamic paraventricular nucleus, Supraoptic nucleus) also play an important role in the control of the extracellular compartment composition and volume. The gigantocellular depressor area (GiDA) is likely to be another vasomotor center involved in blood flow adjustments through direct projections to Preganglionic Sympathetic Neurons (SPN). How GiDA mediates its vasodepressor effects is unknown.

In the last 10 years we have been studying the pathways and neural mechanisms related to muscle and visceral blood flow regulation. Obtained data are not compatible with the proposed model.

Keywords: Bood Pressure. NTS. RVL. CVL.

\section{REFERÊNCIAS}

1 - Feldberg W, Guertzenstein PG. Vasodepressor effect of pentobarbitone sodium. J Physiol (Lond) 1972; 224: 83-8.

2. Guertzenstein PG, Lopes OU. Cardiovascular-responses evoked from the nicotine-sensitive area on the ventral surface of the medulla-oblongata in the cat. J Physiol (Lond) 1984; 347: 345-60.
3 - Guertzenstein PG. Blood-pressure effects obtained by drugs applied to ventral surface of brain-stem. J Physiol (Lond) 1973; 229: 395-408.

4 - Guertzenstein PG, Silver A. Fall in blood-pressure produced from discrete regions of ventral surface of medulla by glycine and lesions. J Physiol (Lond) 1974; 242: 489-4.

5. Colombari E, Sato MA, Cravo SL, Bergamaschi CT, Campos $\mathrm{RR}$, Lopes OU. Role of the medulla oblongata in hypertension. Hypertension 2001; 38: 549-4. 
6 - Timo-laria C. Fisiologia do sistema nervosa. In: Aires MM Fisiologia básica. Rio de Janeiro: Guanabara Koogan; 1995. p. $163-300$.

7 - Yardley CP, Hilton SM. The hypothalamic and brain-stem areas from which the cardiovascular and behavioral components of the defense reaction are elicited in the rat. J Auton Nerv Syst 1986; 15: 227-44.

8 - Yardley CP, Hilton SM. Vasodilatation in hindlimb skeletalmuscle evoked as part of the defense reaction in the rat. $\mathrm{J}$ Auton Nerv Syst 1987; 19: 127-36.

9 - Cravo SL, Possas OS, Ferreira-Neto ML. Rostral ventrolateral medulla: An integrative site for muscle vasodilation during defense-alerting reactions. Cell Mol Neurobiol 2003; 23: 579-95.

10 - Ferreira-Neto ML, Possas Os, Lopes OU, Cravo SL. Evidence for a role of nitric oxide in hindlimb vasodilation induced by hypothalamic stimulation in anesthetized rats. An Acad Bras Cienc 2005; 77: 245-57.

11 - Davisson RL, Possas OS, Murphy SP, Lewis SJ. Neurogenically derived nitrosyl factors mediate sympathetic vasodilation in the hindlimb of the rat. . Am J Physiol Heart Circ Physiol 1997; 41: H2369-76.

12 - Dale N, Gourine AV, Llaudet E, Bulmer D, Thomas T, Spyer KM. Rapid adenosine release in the nucleus tractus solitarii during defence response in rats: real-time measurement in vivo. J Physiol (Lond) 2002; 544: 149-60.

13 - Stlambert JH, THOMAS T, Burnstock G, Spyer KM. A source of adenosine involved in cardiovascular responses to defense area stimulation. Am J Physiol Regul Integr Comp Physiol 1997; 41: R195-200.

14 - Scislo TJ, O'leary DS. Differential control of renal vs. adrenal sympathetic nerve activity by NTS $A(2 a)$ and $P-2 x$ purinoceptors. Am J Physiol Heart Circ Physiol 1998; 44: H2130-9.

15. Scislo TJ, Kitchen AM, Augustyniak RA, O'leary DS. Differential patterns of sympathetic responses to selective stimulation of nucleus tractus solitarius purinergic receptor subtypes. Clin Exp Pharmacol Physiol 2001; 28: 120-4.

16 - Barraco RA, Oleary DS, Ergene E, Scislo TJ. Activation of purinergic receptor subtypes in the nucleus tractus solitarius elicits specific regional vascular response patterns. J Auton Nerv Syst 1996; 59: 113-24.

17 - Ralevic V, Burnstock G. Receptors for purines and pyrimidines. Pharmacol Rev 1998; 50: 413-92.

18 - Kanjhan R, Housley GD, Burton LD, Christie DL, Kippenberger A, Thorne PR, Luo L, Ryan AF. Distribution of the P2X(2) receptor subunit of the ATP-gated ion channels in the rat central nervous system. J Comp Neurol 1999; 407: 11-32.

19 - Stlambert JH, Dawidmilner MS, Silva Carvalho L, Spyer KM. Action of adenosine receptor antagonists on the cardiovascular-response to defense area stimulation in the rat. $\mathrm{Br} \mathrm{J}$ Pharmacol 1994; 113: 159-64.

20 - Thomas T, St Lambert JH, Dashwood MR, Spyer KM. Localization and action of adenosine $\mathrm{A}(2 \mathrm{a})$ receptors in regions of the brainstem important in cardiovascular control. Neuroscience 2000; 95: 513-8.
21 - Tuyau M, Hansen MJ, Dampney RAL, Balcar VJ, Bennett MR Autoradiography of [H-3]alpha,beta-methylene-ATP binding sites in medulla oblongata and spinal cord of the rat. Neurochem Int 1997; 30: 159-69.

22 - Stlambert JH, Dashwood MR, Spyer KM. Role of brainstem adenosine $A(1)$ receptors in the cardiovascular response to hypothalamic defence area stimulation in the anaesthetized rat. Br J Pharmacol 1996; 117: 277-82.

23 - Aicher SA, Reis DJ, Ruggiero DA, Milner TA. Anatomical characterization of a novel reticulospinal vasodepressor area in the rat medulla oblongata. Neuroscience 1994; 60: 761-79.

24 - Aicher AS, Reis DJ. Gigantocellular vasodepressor area is tonically active and distinct from caudal ventrolateral vasodepressor area. Am J Physiol 1997; 272: R731-42.

25. Aicher SA, Reis DJ, Nicolae R, Milner TA. Monosynaptic projections from the medullary gigantocellular reticular formation to sympathetic preganglionic neurons in the thoracic spinal cord. J Comp Neurol 1995; 363: 563-80.

26 - Aicher SA. The gigantocellular depressor area revisited. Cell Mol Neurobiol 2003; 23: 479-90.

27 - Aicher SA, Drake CT. Clonidine evokes vasodepressor responses via alpha(2)-adrenergic receptors in gigantocellular reticular formation. J Pharmacol Exp Ther 1999; 289: 68894.

28 - Bousquet P, Feldman J, Bloch R, Schwartz J. The nucleus reticularis lateralis - a region highly sensitive to clonidine. Eur J Pharmacol 1981; 69: 389-92.

29 - Lim HC, Chong OK, Chan SHH. Characterization of alphaadrenoceptors in the nucleus reticularis gigantocellularis involved in the cardiovascular depressant effects of guanabenz in the rat. Neuropharmacology 1988; 27: 243-9.

30 - Punnen S, Urbanski R, Krieger AJ, Sapru HN. Ventrolateral medullary pressor area - site of hypotensive action of clonidine. Brain Res 1987; 422: 336-46.

31 - Morrison SF, Reis DJ. Responses of sympathetic preganglionic neurons to rostral ventrolateral medullary stimulation. Am J Physiol 1991; 261: R1247-56.

32 - Schreihofer AM, Guyenet PG. Identification of $\mathrm{CI}$ nonadrenergic presympathetic neurons (PSNs) of rostral ventrolateral medulla (RVLM) by juxtacellular label of single recorded cells "in vivo" rat. J Comp Neurol 1997; 387:524-36.

33 - Vu ET, Krasne FB. Evidence for a computational distinction between proximal and distal neuronal inhibition. Science 1992; 255: 1710-2.

34 - Stornetta RL, Mcquiston TJ, Guyenet PG. GABAergic and glycinergic presympathetic neurons of rat medulla oblongata identified by retrograde transport of pseudorabies virus and in situ hybridization. J Comp Neurol 2004; 479: 257-70.

35 - Rampon C, Luppi PH, Fort P, Peyron C, Jouvet M. Distribution of glycine-immunoreactive cell bodies and fibers in the rat brain. Neuroscience 1996; 75: 737-55.

36 - Chen T, Dong YX, Li YQ. Fos expression in serotonergic neurons in the rat brainstem following noxious stimuli: an immunohistochemical double-labelling study. J Anat 2003; 203: $579-88$ 
37 - Jones BE, Holmes CJ, Veiga ER, Mainville L. Gaba-synthesizing neurons in the medulla - their relationship to serotonin-containing and spinally projecting neurons in the rat. $\mathrm{J}$ Comp Neurol 1991; 313: 349-67.

38 - Llewellyn-Smith lj, Martin CL, Minson JB. Glutamate and GABA content of calbindin-immunoreactive nerve terminals in the rat intermediolateral cell column. Auton Neurosci 2002; 98: $7-11$

39 - Rosa D, Ferreira-Neto ML, Cravo SL. Hypertension and increased sympathetic nerve activity induced by excitation/ lesions of the gigantocellular depressor area are not affected by rostral ventrolateral medulla blockade. Auton Neurosci 2005; 119: A136.

40 - Blessing WW, Jaeger CB, Ruggiero DA, Reis DJ. Hypothalamic projections of medullary catecholamine neurons in the rabbit: a combined catecholamine fluorescence and HRP transport study. Brain Res Bull 1982; 9: 279-86.

41 - Saper CB, Reis DJ, Joh T. Medullary catecholamine inputs to the anteroventral third ventricular cardiovascular regulatory region in the rat. Neurosci Lett 1983; 42: 285-91.

42 - Sawchenko PE, Swanson LW. Central noradrenergic pathways for the integration of hypothalamic neuroendocrine and autonomic responses. Science 1981; 214: 685-7.
43 - Colombari DS, Cravo SL. Effects of acute AV3V lesions on renal and hindlimb vasodilation induced by volume expansion. Hypertension 1999; 34:762-7.

44 - Colombari DS, Colombari E, Lopes OU, Cravo SL. Afferent pathways in cardiovascular adjustments induced by volume expansion in anesthetized rats. Am J Physiol Regul Integr Comp Physiol 2000; 279: R884-890.

45 - Pedrino GR, Sera CTN, Cravo SL, Colombari DS. Anteroventral third ventricle lesions impair cardiovascular responses to intravenous hypertonic saline infusion. Auton Neurosci 2005;117: 9-16.

46 - Sera CTN, Colombari DS, Cravo SL. Afferent pathways involved in the renal vasodilation induced by hypertonic saline. Hypertension 1999; 33:1315.

47 - Morris M, Alexander N. Baroreceptor influences on plasma atrial natriuretic peptide (ANP): sinoaortic denervation reduces basal levels and the response to an osmotic challenge. Endocrinology 1988; 122: 373-5. 